\title{
La ciudad contemporánea: Identidad y patrimonio
}

\author{
The contemporary city: Identity and heritage
}

DOI: $10.46932 / \mathrm{sfjdv2n4-089}$

Received in: March 1st, 2021

Accepted in: May 30th, 2021

\section{Irving Samadhi Aguilar Rocha \\ Doctora en Filosofía}

Centro de Interdisciplinar de Investigación en Humanidades del Instituto de Investigación en Humanidades y Ciencias Sociales. Universidad Autónoma del Estado de Morelos. Av. Universidad 1001, Chamilpa. Cuernavaca, Morelos, México, CP. 62209

E-mail: samadhi@uaem.mx

\section{RESUMEN}

Esta reflexión crítica de corte teórico-filosófica toma como objeto de estudio la ciudad y, más específicamente, la transformación que ha sufrido esta idea o conceptualización frente a las nuevas formas de vida, de organización y de control, potencializadas por el desarrollo de la tecnología y la colonización del pensamiento y la vida.

Los habitantes de los pueblos o espacios rurales sufren transformaciones en su estilo de vida con la expansión de la ciudad o mancha urbana, generando la aparición de las formas de vida modernas y tecnológicas que se desarrollan en estas y a las cuales se adaptan. Las prácticas de agricultura poco a poco son desplazadas por las nuevas tecnologías de la urbanización; junto con ello las costumbres y tradiciones se modificarán creando una combinación entre lo urbano y lo rural, poniendo sobre la mesa la discusión en torno a la identidad de los habitantes. Un ejemplo de esto es el poblado de Ocotepec, en el estado de Morelos, cuyo proceso de urbanización resulta más que evidente. Lo interesante de Ocotepec consiste en el hecho de que en el 2014 la UNESCO le otorgó al pueblo el reconocimiento como patrimonio inmaterial cultural por sus costumbres del Día de Muertos, y además de que es considerado pueblo indígena. Esto fue posible porque frente a la expansión de la ciudad y su urbanización se contempló dentro del discurso político de la UNESCO el proteger este patrimonio. De aquí que se vuelva fundamental abordar los temas de la identidad, el patrimonio y las ciudades contemporáneas, caracterizadas como "machas urbanas", lo que abre la reflexión en torno a las dos categorías que nos permitirán aquí analizar éstas: se trata del lugar y del no lugar, de la mano de Marc Augé.

Palabras clave: Ciudad, identidad, patrimonio, lugar, no-lugar.

\begin{abstract}
This philosophical reflection takes the city as the object of study and specifically the transformation of this idea or this concept before the new forms of life, of organization and control, increased by the technological development and the colonization of thought and life.

The inhabitants of a town suffer transformations in their lifestyle with the expansion of a city or an urban spraw, generating new modern and technological ways of life. The agriculture becomes replaced by new technological urbanization and also the ways and traditions change, creating a combination between the country and the urban that questions the identity of their population. As an example of this there is a country called Ocotepec in the state of Morelos, Mexico. The most interesting part of this town is that in 2014 the UNESCO granted Ocotepec the cultural heritage recognition because of the day of the dead, and that it is considered a native country. From this point on becomes fundamental the approach to the subjects
\end{abstract}


of identity, heritage and contemporary cities, also named urban spraws, that open a new reflection in two categories: one being space and the other not space, following Marc Augé proposal.

Key words: City, identity, heritage, space, no space.

\section{INTRODUCCIÓN}

Esta reflexión crítica de corte teórico filosófica toma como objeto de estudio la ciudad y, más específicamente, la transformación que ha sufrido esta idea o conceptualización frente a las nuevas formas de vida, de organización y de control, potencializadas por el desarrollo de la tecnología y la colonización del pensamiento y la vida.

Los habitantes de los pueblos o espacios rurales sufren transformaciones en su estilo de vida con la expansión de la ciudad o mancha urbana, generando la aparición de las formas de vida modernas y tecnológicas que se desarrollan en estas y a las cuales se adaptan. Las prácticas de la agricultura poco a poco son desplazadas por las nuevas tecnologías de la urbanización; junto con ello, las costumbres y tradiciones se modifican creando una combinación, entre lo urbano y lo rural, poniendo sobre la mesa la discusión en torno a la identidad de los habitantes. Un ejemplo de esto es el poblado de Ocotepec, en el estado de Morelos, cuyo proceso de urbanización resulta más que evidente. Lo interesante de Ocotepec consiste en el hecho de que en el 2014 la UNESCO le otorgó al pueblo el estatuto de patrimonio inmaterial cultural por sus costumbres del Día de Muertos, y de que es considerado pueblo indígena. Esto fue posible porque frente a la expansión de la ciudad y su urbanización se contempló dentro del discurso político de la UNESCO el proteger este patrimonio. En la actualidad existe una resistencia por parte de los habitantes originarios de Ocotepec para preservar su identidad, y una de estas formas de resistencia es expresada a través de la apropiación de algunos muros de la calle con producciones artísticas, se trata de murales alusivos a sus costumbres y su historia. Al mismo tiempo, reconocen el desinterés de la juventud por continuar con sus tradiciones. Esto se debe en gran parte a la introducción de la televisión y después de las redes de comunicación (Internet) que transmiten valores y estereotipos que homogenizan las visiones del mundo.

Para el análisis de lo expuesto encontramos necesario utilizar conceptos como lugar y no lugar, ciudad, mancha urbana, identidad, y patrimonio. En este sentido y de manera preliminar, un lugar es entendido como "una realidad simultánea material e inmaterial que asimila el contexto local y también el global" (Neves, 2014, p. 258). Apoyándonos en esta noción, a nuestro parecer, es necesario analizar y comprender la identidad de las ciudades conformadas de esta manera, así como la idea de patrimonio; estas tres ideas son parte de los elementos que permiten la cohesión del tejido social y que, a su vez, permiten hablar de la existencia o de la disolución del espacio urbano público. 
El lugar, por ejemplo, se ha de entender como un espacio significativo, tanto simbólico como cultural, y la identidad junto con el patrimonio son entendidas desde la memoria. Con lo anterior se abre otro hilo conductor de la presente investigación: se trata de pensar las diferencias que existen entre los lugares y los no-lugares en términos de procesos de identificación, como lo presenta Marc Augé, entre el pueblo o las ciudades del pasado y las ciudades contemporáneas. Con el conocimiento obtenido se busca recuperar por un lado, el sentido y el significado del espacio urbano público, de la ciudad, del lugar, del pueblo, y, por el otro lado, se intenta comprender de fondo la disolución del tejido social y sus consecuencias, como por ejemplo la creciente des-humanización de las ciudades.

\subsection{OBJETIVOS}

Los objetivos de este texto consisten en:

1. Esclarecer y analizar conceptos como ciudad, mancha urbana, lugar y no lugar.

2. Comprender la conformación de las ciudades latinoamericanas y de los procesos de expansión del espacio urbano.

\subsection{METODOLOGÍA}

El método de comprensión para el marco teórico es el hermenéutico y con clara influencia fenomenológica. También se utilizó un estudio de caso en el que se observó, se entrevistó a las personas originarias del lugar obteniendo como evidencias videos-entrevistas y fotografías.

\section{CIUDADES CONTEMPORÁNEAS}

La ciudad constituida a partir de los espacios públicos, privados, políticos y artísticos expresa las formas de vida, que, a su vez, se concretan en una cultura. Estos espacios están constituidos por relaciones o articulaciones que propician verdaderos lugares de contenido, lugares que se ven diluidos o ausentes en las sociedades contemporáneas en las que la orientación y el sentido de una vida en común escapa por la evidente cosificación humana, influenciados además por el sistema económico y tecnológico establecido en todo el mundo. La filosofía contemporánea con orientación fenomenológica ha abordado esta problemática, sobre todo desde el pensamiento de Gaston Bachelard, así como también Martin Heidegger, Hannah Arendt, Emmanuel Levinas, Peter Sloterdijk y Michel de Certeau, entre otros. Desde diferentes campos de estudio tenemos a Marc Augé, Geddes y Le Corbusier, que abordan también la cuestión del espacio.

En este sentido, es necesario saber dónde están los seres humanos, lo que nos da la posibilidad de pensar quiénes somos. En esto consiste la importancia de abordar el lugar, la ciudad, el espacio público 
urbano, espacios donde viven las personas. "Mientras la banalidad sella la inteligencia, los hombres no se interesan por su lugar, que parece algo dado; fijan su pensamiento en los fuegos fatuos que les rondan la cabeza en forma de nombres, identidades y negocios" (Sloterdijk, 2003, p. 36).

En la esfera social moderna o en el tejido social moderno priva la falta de relaciones o, mejor dicho, el lazo social o la relación social se convirtió en un artefacto estandarizado, como afirmará Bourriaud:

"En un mundo regulado por la división del trabajo y la ultra especialización, por el devenirmáquina y la ley de la rentabilidad, es importante para los gobernantes que las relaciones humanas estén canalizadas hacia las desembocaduras previstas y según ciertos principios simples, controlables y reproducibles" (Bourriaud, 2008, pp. 7-8).

Se trata, pues, de la disciplinarización y el control del sujeto y de sus relaciones sociales, mecanismos que predeterminan el comportamiento y los efectos en toda relación social e íntima, que generan espacios controlados y expresados en su dimensión objetiva o calculable.

La modernidad política, como lo explica Bourriaud, se produce con la filosofía Ilustrada basada en la voluntad de autonomía de los individuos y de los pueblos, de modo que el progreso de las técnicas y de las libertades, el retroceso de la ignorancia, la mejora de las condiciones de trabajo, debían liberar a la humanidad y permitir una sociedad mejor. Pero fueron, principalmente, los horrores de las dos guerras mundiales las que hicieron que el mito del progreso se derrumbara; en lugar de la emancipación del hombre, el desarrollo de las técnicas y de la razón posibilitó, a través de una racionalización del proceso de producción, la explotación del planeta, incluyendo al propio ser humano, así como la utilización de técnicas de sometimiento cada vez más sofisticadas.

Así pues, los espacios generados desde este pensamiento moderno y que dio lugar al capitalismo, no permiten la vinculación con los seres humanos y el mundo. Esto tiene como consecuencias la psicosis en los individuos y en las sociedades. Los espacios así entornados dejan de ser habitables. De esta forma llegamos al siguiente concepto que es necesario abordar: el habitar. Este está estrechamente relacionado con el concepto de lugar. Habitar parte de la idea bachelardiana y heideggeriana en el sentido de que se refiere al vínculo primigenio (pre-representacional) entre el yo íntimo y el mundo; tiene su fundamento en un espacio vivencial o esfera y es configurador de identidad.

Hay que entender la identidad de forma crítica, distinta de la manera en que tradicionalmente se expresa. Pensarla así permite entenderla como algo cerrado, es decir, la identidad se construye por el Otro, entender el espacio humano abierto desde aquí permite mostrar que es este el que recibe al Otro, es lugar de acogida en el que se puede construir identidad. La casa es condición de posibilidad de identificación y de construcción de un mundo, "para ser un hombre cosmopolita hay que empezar por ser aldeano" 
(Esquirol, 2005, p. 30). La importancia de ello radica en la posibilidad esencial de la interculturalidad, pero no desde la diversidad cultural institucional; el inter de la palabra interculturalidad es mucho más esencial que el mero encuentro entre culturas.

El discurso sobre la ciudad muestra cómo diversas subjetividades reconfiguran, mediante prácticas de resistencia, el espacio social: las calles, las plazas, los ámbitos laborales, los centros comerciales, etc. Las subjetividades se tejen siempre en tensión con las instituciones del Estado, que han de negociar en una sociedad cada vez más compleja y difícil de analizar bajo las categorías de Estado y sociedad. La metáfora del tejido fue utilizada por primera vez por Platón en el "Político", para utilizarlo como el modo del arte de gobernar a la ciudad: se trata de la técnica de tejer y quien la posee es el tejedor. De esta forma se busca ejemplificar diversos aspectos epistemológicos, éticos y políticos.

Ahora bien, partimos de la idea de que la ciudad es la manifestación espacial de una organización social. La ciudad, entendida como espacio urbano y como la sociedad que la conforma, elementos que están íntimamente y necesariamente unidos en el estudio de la primera. Existen dos propuestas interesantes y ricas que abren el análisis y la reflexión sobre ellas; la primera es la de Patrick Geddes, quien propone pensar la ciudad o el espacio urbano como un cáncer, es decir como una mancha. Así como el cáncer es un proceso maligno, en el cual las células pierden sus mecanismos de control ocasionando un crecimiento sin regulación, de la misma manera se entiende la expansión de la ciudad. Una expansión que no permite la diferenciación de espacios, en especial entre el espacio urbano y el espacio rural. En esta expansión todo se vuelve homogéneo, invadiendo los tejidos locales; siguiendo con la metáfora del cáncer se puede decir que se produce una metástasis.

El tránsito de pensar el espacio público como ciudad a pensarlo como mancha urbana se ve reflejado ya en las experiencias, por ejemplo, de Charles Baudelaire, Virginia Woolf y Franz Kafka, que narraban lo inquietantes y hostiles que se presentaban las ciudades. La geografía urbana se transforma en un laberinto que representa la creciente inseguridad, ansiedad y neurosis del individuo ante el espacio urbano así concebido. El sentido del espacio urbano no radica solamente en la forma, sino en las relaciones que rigen la producción y la apropiación de espacios, como lo propone Heidegger, de hecho, es así como establecemos el contenido cultural.

\section{OCOTEPEC, PATRIMONIO Y CIUDAD (MEMORIA)}

La ciudad es, sin duda alguna, lugar de construcción no sólo de identidades sino también de sociedades, en tanto que se crean sentidos y orientaciones para la vida y modos del ser humano que se ven expresadas en las relaciones sociales, en las leyes, en las costumbres, y que cambian constantemente. El modo de vida en las diferentes ciudades refleja un modo particular de ser y pensar, en especial en las 
sociedades contemporáneas con sus grandes urbes, que a través del desarrollo de las tecnologías dejan ver algo paradójico en la conformación de lugares para el hombre. Lo paradójico surge cuando estos espacios lejos de ser lugares para el desenvolvimiento humano, crean no-ciudades o "manchas urbanas" que no tienen relación con aquél, lugares impersonales que Marc Augé llama los no lugares. Este es el caso del pueblo de Ocotepec en el estado de Morelos, que en la actualidad es considerado como una colonia de la ciudad de Cuernavaca pero que en realidad es un pueblo que fue absorbido por la zona urbana. El pueblo esta subdividido en cuatro barrios: La Candelaria (Tlaneui), Dolores (Culhuakan), Ramos (Tlakopan) y Santa Cruz (Xalxokotepeazola). En el pasado fue un pueblo indígena cuya lengua materna, y que aún se sigue escuchando por las calles, era el náhuatl. La urbanización que ha empezado a tener este pueblo es evidente, pero en el 2014 la UNESCO reconoció sus costumbres de Día de Muertos como patrimonio inmaterial cultural. Esto es lo que se mantiene vivo, la costumbre y tradición de este poblado, pues es la gran expansión de la urbanización, la amenaza de la mancha, lo que hizo entrar al discurso político de la UNESCO para proteger este patrimonio. Antes de recibir la distinción de la UNESCO ya existía -y todavía existe- la preocupación de los habitantes originarios del pueblo. A través de las entrevistas realizadas a éstos, expresaron la importancia de las tradiciones y costumbres del pueblo, así como su creciente preocupación por los jóvenes del pueblo debido al hecho de estar perdiendo estos legados que les dejan sus familias. Reconocen que fue la inserción primero de la televisión y después de las redes de comunicación, sobre todo el Internet, lo que posibilitó y posibilita el desarraigo de la tradición en el momento en que los jóvenes aprenden y asimilan otros valores, formas de vida que corresponden a la industria cultural, dejando a un lado o sin valor las costumbres del lugar donde habitan.

Sin embargo, sostenemos que la idea de la ciudad se ha de entender como el entramado que permite crear nuevas relaciones sociales, nuevas leyes o nuevas costumbres que permitan la reconfiguración constante de espacios que posibiliten la experiencia rica en significación e identidad, y en este sentido generan cohesión social en el momento en que existe por parte de los habitantes originarios el interés por preservar su cultura independientemente de que haya sido reconocida una de sus tradiciones más arraigadas; también hemos comprobado que las ciudades digitales y los espacios de anonimato impiden tanto la significación como la identidad, porque homogenizan y transmiten los mismos patrones de pensamiento y acción.

Al parecer, la preocupación ante la pérdida de identidad y de cohesión social es evidente; una de las formas que tienen de expresarlo los habitantes de Ocotepec es a través de la apropiación de espacios públicos urbanos como son los muros. En ellos se ven reflejados temas sobre su identidad y su historia. Se trata de la relación que hay entre el sentido de vida de la población -que ha de ser considerada ya un 
espacio urbano aunque para los pobladores no sea así- y la sensibilidad de los habitantes. Algunos ejemplos de lo expuesto se reflejan en las siguientes imágenes.

\section{A MODO DE CONCLUSIÓN}

La expansión de la mancha urbana no solo disuelve la identidad y con ello el tejido social al no haber un interés en común, sino que se ve también reflejada, y quizás menos en el tipo de poblaciones como Ocotepec, en la velocidad en la que se vive o en el flujo de coches y personas. En el caso de Ocotepec, encontramos su calle principal, que comunica a los "lugares de encuentro", como conflictiva y con exceso de autos; aquí es imposible detenerse por un helado, fruta o incluso ir a la iglesia. Con ello, hemos de preguntarnos qué es una ciudad y qué no lo es. Como hemos sostenido más arriba, la ciudad sería un lugar en donde fuese posible el encuentro entre diferentes personas y diferentes culturas, un lugar de relaciones que permitieran la vida en común. Pero una no ciudad para nosotros equivaldría a lo que Geddes consideraba "mancha urbana", mancha que no permite la diferenciación de tejido, que se extiende homogenizando-colonizando vida y pensamiento. Esta enfermedad trae consecuencias, altera al cuerpo, altera a las sociedades, no solo rurales sino también a las zonas urbanas, las cambia y modifica bajo la lógica capitalista. Los grandes monopolios comerciales y las tecnologías que permiten que sea más eficaz la expansión.

La ciudad tendría que ver más con el sentido ontológico del espacio humano, como ya se lo señalaba desde las investigaciones fenomenológicas heideggerianas, al preguntarse por el dicho sentido necesario para el habitar, para la existencia ontológicamente humana; se trata de entender el lugar o espacio como existencial, vivido, afectivo y corpóreo, no el espacio físico-matemático -maquinal, si se quiere decir así-, es decir, donde los horarios y los trayectos están establecidos por el ritmo y el flujo inevitable de la velocidad. Lo anterior nos presenta otra perspectiva de la ciudad contemporánea, nos abre el ámbito de reflexión desde la práctica, la apropiación y la comprensión del lugar desde la crítica. En las no ciudades, o mancha urbana, no hay lugar para la identidad. Se trata de pensar la ciudad como un lugar de memoria y de relaciones simbólicas, que son fundamentales para el arraigo, lo que los ocotepeños defienden y que ven desaparecer en la vida cotidiana.

El periódico El País publicó un artículo titulado "Pensar la ciudad como un pueblo". El título puede abrir un debate entre los conceptos de ciudad y pueblo, pero este texto no está pensando a la ciudad en los términos de un pueblo con características rurales, sino más bien en términos de la población, es decir, en que en la ciudad habitan pobladores, aludiendo a la pérdida de hogar -nosotros decimos del lugar-donde sea posible el habitar humano. 
Cierre los ojos. Imagine el lugar en el que vive ahora dentro de 40 años. ¿Qué ve? 'Autopistas a varias alturas'. 'Rascacielos gigantes'. 'Puentes que conectan bloques'. 'Pantallas por todas partes'. 'Atascos eternos'. Las respuestas entroncan con el crecimiento desmesurado, los problemas actuales y la perspectiva de la metrópolis. A miles de kilómetros de los deseos y las previsiones de urbanistas, arquitectos y expertos en desarrollo y planificación. A miles de kilómetros de lo que debería ser concebido un hogar. Hay que pensar en las ciudades como el pegamento urbano que une la individualidad." (Valdés, 2015)

La sobrepoblación implica también la expansión inevitable, generando nuevas construcciones urbanas, así como el gran crecimiento de los centros comerciales en pos del progreso. La mancha urbana se expande y devora no solo al hombre rural sino a todo modo de vida humana, comunidades, sociedades, incluso el hábitat natural. El único recordatorio que queda de los pueblos es el recuerdo de lo que existió ahí, el lugar legendario o el objeto legendario, hoy llamado "patrimonio".

Estas antiguallas que parecen dormir, casas desfiguradas, fábricas que ahora tienen nuevos destinos, vestigios de historias hundidas en algún naufragio, hoy todavía erigen las ruinas de una ciudad desconocida, extranjera. Irrumpen en la ciudad modernista, masiva, homogénea, como un lapsus de un lenguaje desconocido tal vez inconsciente. [...] Las cosas viejas se hacen notables. Lo fantástico se encierra ahí, en lo cotidiano de la ciudad. Se trata de un aparecido que atormenta desde ahora el urbanismo. Naturalmente, lo fantástico no volvió por sí sólo. Lo trajo la economía proteccionista. [...] Este aparecido se exorciza bajo el nombre de "patrimonio". (De Certeau, 1999: pp. 135-136)

Los habitantes originarios de pueblos y barrios urbanos viven la expansión como una experiencia de pérdida en las relaciones con los demás, en el paisaje, en sus costumbres, en la seguridad. A partir de aquí cabría la pregunta que ya Massimo Cacciari se planteaba acerca de si es posible habitar sin lugar:

¿Es posible vivir sin lugar? ¿Es posible habitar allí donde no se producen lugares? El habitar no se produce allí donde se duerme de vez en cuando, donde se mira la televisión y se juega con el ordenador personal; el lugar del habitar no es el alojamiento. Sólo una ciudad puede ser habitada, pero no es posible habitar la ciudad si ésta no se dispone para el habitar: es decir, si no "proporciona" lugares. (Cacciari, 2010: 35)

Siempre se ha pensado que donde hay ciudades se puede habitar, pero esta proposición es falsa. Ya lo ha dicho Massimo: se necesitan lugares para habitar, esto es, lugares donde sea posible el encuentro, la seguridad, el intercambio y la acción. Habitar, en el sentido ontológico, tiene dos direcciones: una en tanto la necesidad al interior del permanecer amparado, y otra como un deseo en el hecho de morar, como un de-morarse en la protección. Se trata de una relación de cercanía y familiaridad entre el hombre y el mundo y por lo que es posible espaciar una habitación o un lugar. En las ciudades contemporáneas más bien se tiene la distancia o la pérdida propia del exiliado, el estar en el ir y venir, entre la huida y el permanecer, esto expresa la búsqueda de una patria-casa desconocida que aparece como figura imposible y que remite al lugar de nacimiento, a lo natal. En este sentido, lo natal es la expresión en acto, esa sensación de no estar, propia de aquellos que buscan un lugar para habitar. 


\section{REFERENCIAS}

Bachelard, G. (2005). La poética del espacio. México. FCE.

Bourriaud, N. (2008). Estética relacional. Buenos Aires. Adriana Hidalgo.

Cacciari, M. (2010). La Ciudad [trad. de Moisés Puente]. Barcelona. Gustavo Gili.

De Certeau, M. (1999). La invención de lo cotidiano [trad. de Alejandro Pescador]. México. Universidad Iberoamericana.

Esquirol, J. M. (2005). Uno mismo y los otros. Barcelona. Herder.

Geddes, P. (1997). "Metropolis Unbound. The Sprawling American City and the Search for Alternative". En The American Prospect, núm. 35. Cambridge, Massachusetts.

Heidegger, M. (2003). Ser y tiempo. Madrid, Trotta. (2001). Conferencias y artículos. Barcelona, Serbal.

Neves, V. (2014). "Los espacios públicos: vacíos con identidad". En Identidad y espacio público. Barcelona. Gedisa.

Sánchez González, D. y Domínguez Moreno, L. (coords.) (2014). Identidad y espacio público. Barcelona. Gedisa.

Silva, A. (2006). Imaginarios Urbanos. Colombia. Arango Editores.

Sloterdijk, P. (2003). Esferas I. Madrid. Siruela.

Valdés, I. (2015). "Pensar la ciudad como pueblo". En El País. Madrid, 07 de Octubre 2015. http://elpais.com/elpais/2015/09/30/planeta_futuro/1443622395_272649.html/

Vargas Rodríguez, A. (coord.) (2011). Ciudad contemporánea. Colombia. Universidad de Bogotá.

Ejidos Urbanizados de Cuernavaca I/IV. (2008). Push \& Play producciones (televisora independiente) con el apoyo del Ayuntamiento de Cuernavaca, Regiduría de Desarrollo Agropecuario. Disponible en línea: <http://www.pushandplay.org>: http://www.youtube.com/watch?v=YIEuVevovpw/ 